\title{
Enhanced functionality of cantilever based mass sensors using higher modes and functionalized particles
}

Dohn, Søren; Sandberg, Rasmus Kousholt; Svendsen, Winnie Edith; Boisen, Anja

Published in:

The 13th International Conference on Solid-State Sensors, Actuators and Microsystems, 2005. Digest of Technical Papers. TRANSDUCERS '05.

Link to article, DOI:

10.1109/SENSOR.2005.1496497

Publication date:

2005

Document Version

Publisher's PDF, also known as Version of record

Link back to DTU Orbit

Citation (APA):

Dohn, S., Sandberg, R. K., Svendsen, W. E., \& Boisen, A. (2005). Enhanced functionality of cantilever based mass sensors using higher modes and functionalized particles. In The 13th International Conference on SolidState Sensors, Actuators and Microsystems, 2005. Digest of Technical Papers. TRANSDUCERS '05. (Vol. 1, pp. 636-639). IEEE. https://doi.org/10.1109/SENSOR.2005.1496497

\section{General rights}

Copyright and moral rights for the publications made accessible in the public portal are retained by the authors and/or other copyright owners and it is a condition of accessing publications that users recognise and abide by the legal requirements associated with these rights.

- Users may download and print one copy of any publication from the public portal for the purpose of private study or research.

- You may not further distribute the material or use it for any profit-making activity or commercial gain

- You may freely distribute the URL identifying the publication in the public portal 


\title{
ENHANCED FUNCTIONALITY OF CANTILEVER BASED MASS SENSORS USING HIGHER MODES AND FUNCTIONALIZED PARTICLES
}

\author{
Søren Dohn, Rasmus Sandberg, Winnie Svendsen, and Anja Boisen \\ Department of Micro and Nanotechnology, Technical University of Denmark, DK-2800 Lyngby \\ e-mail: sd@mic.dtu.dk
}

\begin{abstract}
By positioning a single gold-particle at different locations along the length axis on a cantilever based mass sensor, we have investigated the effect of mass position on the mass responsivity and compared the results to simulations. A significant improvement in quality factor and responsivity was achieved by operating the cantilever in the $4^{\text {th }}$ bending mode thereby increasing the intrinsic sensitivity. It is shown that the use of higher bending modes grants a spatial resolution and thereby enhances the functionality of the cantilever based mass sensor.
\end{abstract}

Keywords: Cantilever, Sensor, Sensitivity, Modes

\section{INTRODUCTION}

The demand for sensors with high sensitivity, improved functionality and reduced analyte usage as well as the demand for portable devices is driving the development of sensors from the micro- to the nanoregime. Cantilever based sensors have been a part of this development since the method was proposed by Chen et. al [1] in 1995. Today cantilevers are used widely in sensor applications in the static mode where a reaction on the surface creates surface stress which in turn causes a bending of the cantilever [2-3]. Also, the use of vibrating cantilevers shows great promises for

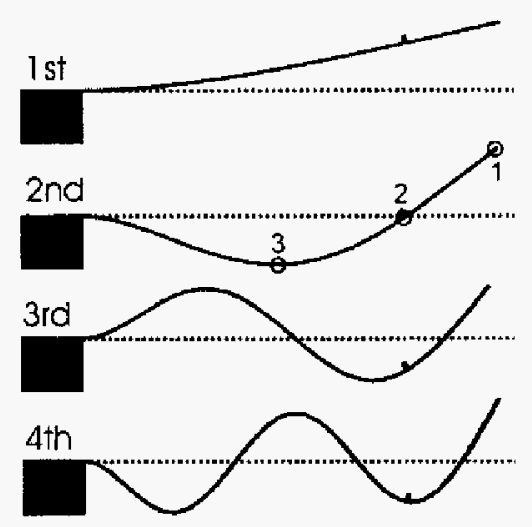

Fig. 1 Schematics of the first four bending modes of a cantilever seen from the side. Due to the nodal points certain parts of the cantilever do not take part of the vibration, and the particle depicted approximately $3 / 4$ from the base of the cantilever is in theory not detectable in the second mode. sensor applications and recently systems capable of detecting masses in the $10^{-18} \mathrm{~g}$ range have been reported [4-5]. Here we report on the investigation of cantilever sensitivity using a movable point-mass, and experimental results are compared with simulations.

The resonant frequencies for a cantilever are given by

$f_{n}=\omega_{n} / 2 \pi=(2 \pi)^{-1} \sqrt{k / m_{n}^{*}}$

where $m_{n}^{*}$ is the effective mass of the $n^{\text {th }}$ mode of vibration. The higher the mode of vibration the smaller the effective mass due to the increasing number of nodal points. Schematics of the first four bending modes are shown in fig. 1 . It is seen that certain regions of the cantilever do not take part in the vibration and the positions of these are changing with the mode of vibration. The resonant frequency change caused by added mass therefore depends on the mass position.

This has previously been investigated theoretically on the macroseopic scale [6-8]. The sensitivity of cantilever based mass sensors has been investigated by several groups [9-10] and the terminology used here is adopted from the work of Ekinci et al. [10]. The mass responsivity is $R \approx 2 \pi \Delta f / \Delta m$, and the intrinsic sensitivity is $\delta m_{n} \propto R^{-1} \sqrt{1 / \omega_{n} Q_{n}}$ (assuming the energy in the cantilever is constant).

As an alternative to use the entire cantilever surface for molecular adsorption we propose to use only certain areas of the cantilever and positioning these for optimum sensitivity. We investigate the concept by adding a point-mass to a micro cantilever. By moving this point-mass in small steps along the length direction of the cantilever, and simultaneously determining the resonant frequency for several bending modes, we can locate the positions yielding high mass responsivity. The experimental data is compared to simulations performed using finite element software.

\section{EXPERIMENT}

The experimental setup (fig. 2) consists of a HeNe laser, which is focused on a micrometer sized cantilever. The cantilever-chip is clamped on top of a piezo actuator which is driven by a HP4194A gain/phase analyzer and placed in a custom built vacuum chamber capable of maintaining a vacuum of 0.5 mbar at which all experiments are performed. The position of the reflected beam is registered by a photo- 


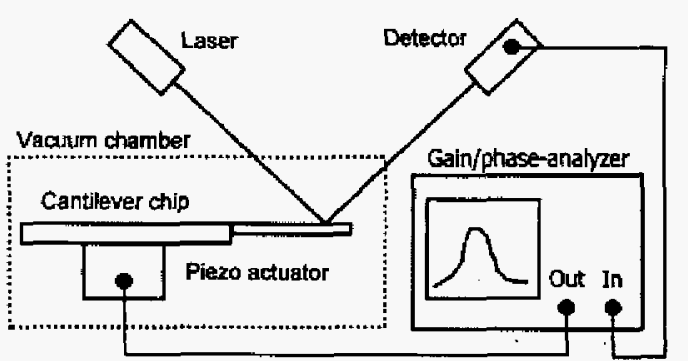

Fig. 2 Schematic of the setup used in the experiments. The micro cantilever chip is clamped on a piezo actuator driven by a Gain/Phase analyzer. Both cantilever and piezo actuator are placed in a vacuum chamber. $A$ laser is focused on the cantilever and the position of the reflected beam is detected by a photodiode, and the signal is feed into the gain/phase analyzer.

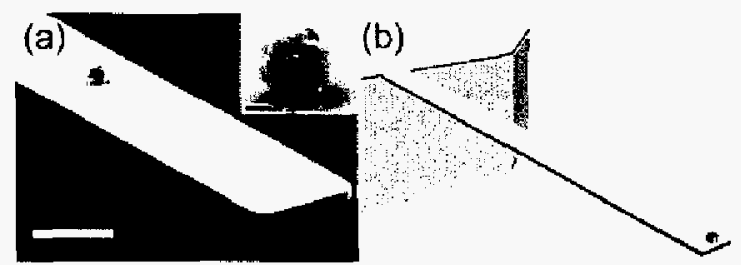

Fig. 3 (a) SEM image showing part of the micro cantilever with a gold-bead positioned close to the apex. The scale bar is $5 \mu \mathrm{m}$. The inset shows a closeup of the gold particle (Scale bar $500 \mathrm{~nm}$ ). (b) Simulated structure used for calculation of the theoretical shift in resonance as a function of mass position. The simulated cantilever has the same dimensions as the cantilever used in the experiment, and the mesh generated for the calculations consists of bricks which are $1 \mu \mathrm{m}$ in the length direction of the cantilever.

diode, and the signal is after amplification measured using the HP4194A gain/phase analyzer. By actuating the cantilever using a piezo actuator driven by the gain/phase analyzer the amplitude and phase of the resonating cantilever can be detected. Data from the gain/phase analyzer is transferred through a GPIB interface to a PC.

The cantilever used in the experiment is fabricated in $\mathrm{SiO}_{2}$ and subsequently coated on the topside with 10 $\mathrm{nm} \mathrm{Ti}$ and $100 \mathrm{~mm}$ Au (the fabrication process is described in [11]). The cantilever dimensions are measured in a SEM yielding a length, width and thickness of approximately $153 \mu \mathrm{m}, 11 \mu \mathrm{m}$ and 1.05 $\mu \mathrm{m}$ respectively. A rather large cantilever with a large surface area was chosen because it facilitates easy manipulation of particles on the cantilever surface and it gives high resolution in the length direction. The metal coating was used to improve the reflectivity of the $\mathrm{SiO}_{2}$ cantilevers.

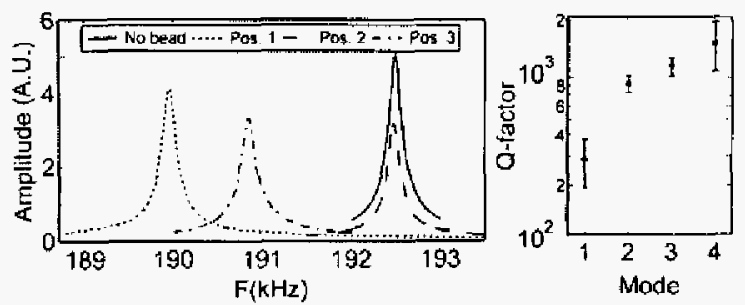

Fig. 4 Resonant peaks for the $2^{\text {nd }}$ bending mode as function of position and the average Q-factor for the recorded bending modes. The positions are indicated in fig. 1 . Only part of the recorded $2^{\text {nd }}$ bending mode peaks are shown for clarity.

A single gold-bead with a radius of $0.9 \mu \mathrm{m}$, corresponding to a mass of approximately $60 \mathrm{pg}$, is positioned on the cantilever (fig. 3a) using a manipulation setup based on a high resolution optical Navitar microscope: A Newport XYZ stage is used for cantilever positioning, while an etched tungsten tip with a tip diameter of roughly $1 \mu \mathrm{m}$ mounted on a Burleigh PCS-5400 piezo-micromanipulator is used for manipulating the gold-bead. The position of the gold bead is determined from optical images with an estimated accuracy of $\pm 1 \mu \mathrm{m}$. After positioning of the gold-bead the resonant frequency of the cantilever is measured, and the bead is pushed with the tungsten tip to a new location where the resonant frequency is measured again.

The cantilever structure on which simulations are performed using the finite element software, CowentorWare, is shown in fig. $3 \mathrm{~b}$. The simulated cantilever has the same dimensions as the cantilever used in the experiment and is also metal coated. The density $(\rho)$ and Young's $(E)$ modulus for the simulated materials are $\rho=2150 \mathrm{~kg} / \mathrm{m} 3$ and $E=70 \mathrm{GPa}\left(\mathrm{SiO}_{2}\right)$, $\rho-4500 \mathrm{~kg} / \mathrm{m} 3$ and $E=110 \mathrm{GPa}$ (Ti) and $\rho=19300$ $\mathrm{kg} / \mathrm{m} 3$ and $E=57 \mathrm{GPa}(\mathrm{Au})$. The mesh generated for the calculations consists of bricks which are $1 \mu \mathrm{m}$ in the length direction of the cantilever. The gold-bead is simulated as a gold-box with sidewalls of $1.8 \mu \mathrm{m}$ in length, and this causes two problems. The mass of the box is too big, and since the contact area between a surface and box is much larger than between a surface and a sphere, a stiffening of the cantilever will occur. Therefore the density of the box is reduced to $\rho=10106$ $\mathrm{kg} / \mathrm{m}^{3}$ so the weight matches that of the gold-bead. Also Young's modulus of the box was reduced to a $100^{\text {th }}$ of that of gold (with $E=0.57 \mathrm{GPa}$ ), since no stiffening was observed at this value.

\section{RESULTS AND DISCUSSION}

The resonant frequency $\left(f_{\mathrm{cxp}}\right)$ of the first four bending modes of the micro-cantilever is measured before the gold-bead is positioned on the cantilever. The results are shown in Table I together with the simulated 
Table 1 The resonant frequency measured and simulated for the first four bending modes of the cantilever together with the experimentally observed maximum change in frequency and relative sensitivity for the specific mode.

\begin{tabular}{lcccc}
\hline Mode & 1 & 2 & 3 & 4 \\
\hline$f_{\mathrm{exp}}(\mathrm{Hz})$ & 30712 & 192493 & 539060 & 1056640 \\
$f_{\mathrm{sim}}(\mathrm{Hz})$ & 31734 & 198815 & 556486 & 1089920 \\
$\Delta f_{\max }(\mathrm{Hz})$ & 480 & 2541 & 6059 & 9864 \\
$\delta m_{1} / \delta m_{n}$ & 1 & 23 & 102 & 276 \\
\hline
\end{tabular}

resonant frequencies $\left(f_{\text {sim }}\right)$. The simulated resonant frequencies are within $3.5 \%$ of the experimental values for all the measured bending modes.

When adding the single gold-bead to the tip of the cantilever the resonant frequency of all four modes decrease as expected from equation 1. Typical examples of the recorded amplitude resonance peaks are depicted in fig. 4. These particular resonant peaks are taken from the $2^{\text {nd }}$ bending mode, and the numbering of the peaks refers to the position of the gold-bead on the cantilever (fig. 1). The fitted peaks of the first four bending modes all have an average standard deviation of less than $0.01 \%$, and the standard deviation of successive experiments with no movement of the bead is within this limit. From measurements at all positions an average $\mathrm{Q}$-factor was calculated for

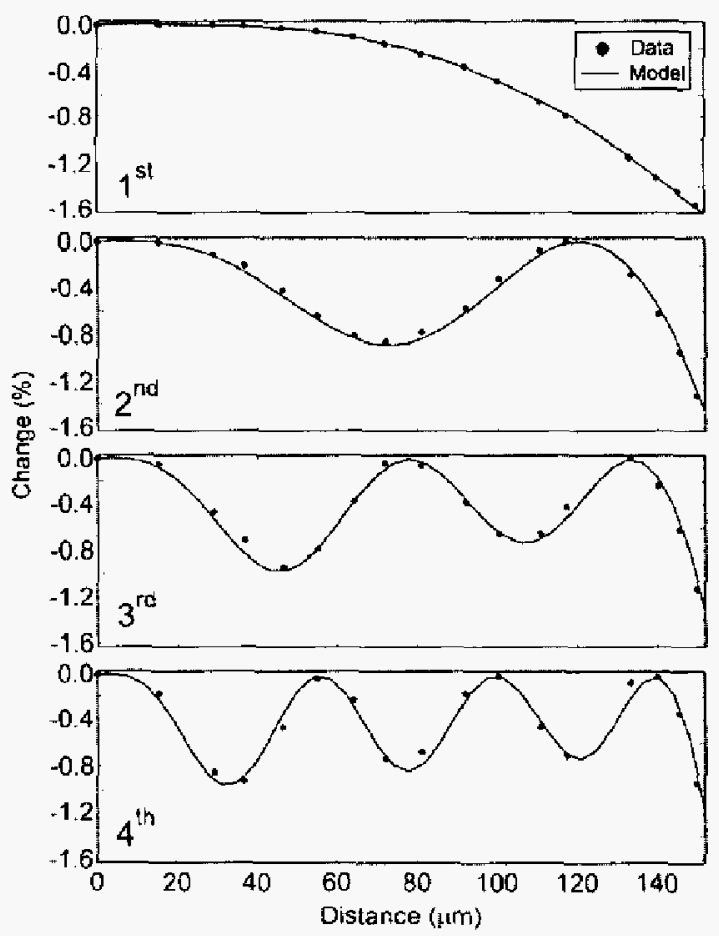

Fig. 5 Measured shift in resonance as a function of position compared to the simulated values shown for the first four bending modes of vibration. each mode (fig. 4). The Q-factor increases almost a factor of 3 going from the first to the second bending mode and the $4^{\text {th }}$ mode has a Q-factor that is more than 5 times larger than the $1^{\text {st }}$ mode. There is no significant difference between the Q-factor of the loaded and unloaded cantilever.

The change in resonance frequency for the first four bending modes of the cantilever as a function of particle position along the length axis has been recorded. The experimentally observed relative change in resonance is compared to the values obtained by simulations and excellent agreement is obtained for all bending modes (fig. 5). For all modes the highest change in frequency $\left(\Delta f_{\max }\right)$ is obtained when the goldbead is positioned at the tip of the cantilever (experimental values listed in Table 1). The absolute change in frequency and thereby the responsivity increases with the mode number giving a mass responsivity of $-5 \mathrm{fg} / \mathrm{Hz}$ in the $4^{\text {th }}$ mode for the added mass of $60 \mathrm{pg}$. The relative sensitivities $\delta M_{1} / \delta M_{n}$ have been calculated (Table I), and the sensitivity is seen to increase by a factor of $\sim 300$ using the $4^{\text {th }}$ mode.

The highest measured resonance mode results in the best mass responsivity of the cantilever and corresponds to our theoretical predictions. The large increase in sensitivity with increasing bending modes is mainly owed to a decrease in the effective mass (eq. 1), but also to the increase in Q-factor. The rather low Q-factor observed for the cantilevers are due to the metal coating [12], and similar uncoated cantilevers have $\mathrm{Q}$-factors that are 10 times higher. The increasing Q-factor has previously been observed [13-14] and is likely due to the smaller centre of mass movement and smaller radiation at the support for increasing bending modes [15].

\section{CONCLUSION}

In conclusion, a mass responsivity of approximately 5 $\mathrm{fg} / \mathrm{Hz}$ has been observed for a micrometer sized cantilever when operating the cantilever in the $4^{\text {th }}$ mode. The increase in Q-factor and resonant frequency as well as the decrease in effective mass makes the intrinsic sensitivity of the cantilever significantly better for increasing mode numbers ( $\sim 300$ for the $4^{\text {th }}$ mode). This implies that a 300 times smaller mass can be detected in the $4^{\text {th }}$ mode compared to the $1^{\text {st }}$, assuming the cantilever is the dominant source of noise. To fully utilize the capabilities of a cantilever based mass sensor it is therefore important to operate it at higher modes.

The experimentally observed changes in resonant frequency when adding a point mass show excellent agreement with the simulations for the first four bending modes. From the measurements it follows that a single cantilever operated in the $4^{\text {th }}$ mode has four positions for high sensitivity mass sensing. The number 
of sensitive positions can be further increased by operating at even higher modes.

The method presented can be used for enhancing the performance compared to conventional cantilever based mass sensors since the Q-factor degrading gold coating of the entire cantilever can be avoided. Using higher bending modes for detection it is possible to achieve a significant increase in the intrinsic sensitivity compared to the fundamental bending mode and moreover the method in principle grants a spatial resolution to the cantilever based mass sensor. By measuring different modes of vibration it should be possible to locate the position of the added mass on the cantilever. One possible application could be to detect more than one specific molecule using a single cantilever, by position several particles or coated areas functionalized for recognition of different molecules individually on the cantilever.

\section{References}

[1] G. Y. Chen, T. Thundat, E. A. Wachter, and R. J. Warmack, "Adsorption-Induced Surface Stress and Its Effects on Resonance Frequency of Microcantilevers", Journal of Applied Physics, 8, 3618-3622, (1995)

[2] J. Fritz, M. K. Baller, H. P. Lang, H. Rothuizen, P. Vettiger, E. Meyer, H. J. Guntherodt, C. Gerber, and J. K. Gimzewski, "Translating biomolecular recognition into nanomechanics", Science, 5464, 316-318, (2000)

[3] P. A. Rasmussen, J. Thaysen, O. Hansen, S. C. Eriksen, and A. Boisen, "Optimised cantilever biosensor with piezoresistive read-out", Ultramicroscopy, 1-4, 37l-376, (2003)

[4] T. Ono, X. X. Li, H. Miyashita, and M. Esashi, "Mass sensing of adsorbed molecules in subpicogram sample with ultrathin silicon resonator", Review of Scientific Instruments, $\mathbf{3}$, 1240-1243, (2003)

[5] K. L. Ekinci, X. M. H. Huang, and M. L. Roukes, "Ultrasensitive nanoelectromechanical mass detection", Applied Physics Letters, 22, 4469-4471, (2004)
[6] S. Park, W. K. Chung, Y. Youm, and J. W. Lee, "Natural frequencies and open-loop responses of an elastic beam fixed on a moving cart and carrying an intermediate lumped mass", Joumal of Sound and Vibration, 3, 591-615, (2000)

[7] M. Chiba and T. Sugimoto, "Vibration characteristics of a cantilever plate with attached spring-mass system", Journal of Sound and Vibration, 2, 237-263, (2003)

[8] K. H. Low, "On the eigenfrequencies for mass loaded beams under classical boundary conditions", Journal of Sound and Vibration, 2, $381-389,(1998)$

[9] P. I. Oden, "Gravimetric sensing of metallic deposits using an end-loaded microfabricated beam structure", Sensors and Actuators $B$ Chemical, 3, 191-196, (1998)

[10] K. L. Ekinci, Y. T. Yang, and M. L. Roukes, "Ultimate limits to inertial mass sensing based upon nanoelectromechanical systems", Journal of Applied Physics, 5, 2682-2689, (2004)

[11] C. L. Petersen, T. M. Hansen, P. Boggild, A. Boisen, O. Hansen, T. Hassenkam, and F. Grey, "Scanning microscopic four-point conductivity probes", Sensors and Actuators A-Physical, 1, 53-58, (2002)

[12] R. H. Blick, A. Erbe, L. Pescini, A. Kraus, D. V. Scheible, F. W. Beil, E. Hoehberger, A. Hoerner, J. Kirschbaum, and H. Lorenz, "Nanostructured silicon for studying fundamental aspects of nanomechanics", Journal of Physics-Condensed Matter, 34, R905-R945, (2002)

[13] L. B. Sharos, A. Raman, S. Crittenden, and R. Reifenberger, "Enhanced mass sensing using torsional and lateral resonances in microcantilevers", Applied Physics Letiers, 23. 4638-4640, (2004)

[14] J. L. Yang, T. Ono, and M. Esashi, "Mechanical behavior of ultrathin microcantilever", Sensors and Actuators A-Physical, 1-3, 102-107, (2000)

[15] G. Stemme, "Resonant Silicon Sensors", Journal of Microelectromechanical Engineering, 113$125,(1991)$ 